\title{
Condensation-induced jumping water drops
}

\author{
R. D. Narhe, ${ }^{1,2, *}$ M. D. Khandkar, ${ }^{3}$ P. B. Shelke, ${ }^{4}$ A. V. Limaye, ${ }^{5}$ and D. A. Beysens ${ }^{2,6, \dagger}$ \\ ${ }^{1}$ Department of Physics and Applied Mathematics, University of Navarra, 31080 Pamplona, Spain \\ ${ }^{2}$ CEA-ESEME, ESPCI-PMMH, 10, rue Vauquelin, 75231 Paris Cedex 05, France \\ ${ }^{3}$ Department of Applied Physics, Helsinki University of Technology (HUT), P.O. Box 1100, FIN-02015 Espoo, Finland \\ ${ }^{4}$ Department of Physics, Ahmednagar College, 414001 Ahmednagar, India \\ ${ }^{5}$ Department of Physics, University of Pune, Pune 411007, India \\ ${ }^{6}$ Service des Basses Températures, CEA-Grenoble, 38054 Grenoble, France \\ (Received 4 February 2009; revised manuscript received 31 March 2009; published 17 September 2009)
}

\begin{abstract}
Water droplets can jump during vapor condensation on solid benzene near its melting point. This phenomenon, which can be viewed as a kind of micro scale steam engine, is studied experimentally and numerically. The latent heat of condensation transferred at the drop three phase contact line melts the substrate during a time proportional to $R$ (the drop radius). The wetting conditions change and a spontaneous jump of the drop results in random direction over length $\sim 1.5 R$, a phenomenon that increases the coalescence events and accelerates the growth. Once properly rescaled by the jump length scale, the growth dynamics is, however, similar to that on a solid surface.
\end{abstract}

DOI: 10.1103/PhysRevE.80.031604

PACS number(s): 68.03.Cd, 68.08.Bc, 64.60.Q-

\section{INTRODUCTION}

Condensation of water vapor on cold surfaces is a daily experience to all of us, e.g., the beautiful patterns of breath figures (condensed water drops) on windowpane or dew on grass blades in the morning. Moreover, the condensation phenomenon has great importance in variety of fields such as heat and mass transfer [1], early stages of thin film growth $[2,3]$, fabrication of ordered micro/nanoporous membranes [4-6], atmospheric water recovery [7] etc. The preliminary research on breath figures dates back to the work done by Aitken, Rayleigh, and Baker [8] during 1893-1922. Many fundamental laws of nucleation and growth dynamics of breath figures are well identified after the early work by Beysens and Knobler [9-12]. However, the spectacular jumping of drops condensing on a solid substrate near its melting point (cyclohexane) $[13,14]$ has been only partially investigated. In this phenomenon mechanical work is released. It thus appears to be a unique example of microsteam engine and per se deserves special attention.

In general, the growth dynamics of breath figures takes place in three important stages. (i) In the initial stage, the surface coverage, $\varepsilon^{2}$, which is the ratio of droplet area to substrate area, is low. The drops are isolated (no coalescence) and grow as $t^{1 / 3}$ ( $t$ is time). (ii) In the intermediate (crossover) stage, $\varepsilon^{2}$ increases rapidly and reaches a maximum value. (iii) The coalescence dominated (self-similar) stage corresponds to a surface coverage that saturates. The average drops radius grows as $t^{1}$. These growth laws were observed and obtained both analytically and in computer simulation studies [15-18].

Coalescence is a key phenomenon to rescale and accelerate growth. Thus, if due to certain mechanism, the coales-

\footnotetext{
*Permanent address: Department of Physics, Fergusson College, Pune, India.

${ }^{\dagger}$ Corresponding author; daniel.beysens@espci.fr
}

cence events are enhanced, the growth dynamics can be deeply modified. This is precisely the case for drops jumping on a solid surface near its melting point. A question naturally occurs whether the jump of drops changes the growth dynamics or not. We address this question in the present experimental and numerical study where water drops are condensing on solid benzene near its melting point.

\section{EXPERIMENTS}

The condensation chamber consists in a Peltier-element thermostat enclosed in a Plexiglas box. A small piece of cleaned silicon wafer of size $1 \times 1 \mathrm{~cm}^{2}$ is fixed on a thick electrolytic copper plate by means of a thin film of water in order to ensure a good thermal contact. A small drop ( $\approx 20 \mu \mathrm{l}$ ) of liquid benzene (Sigma Aldrich, 99.99\% pure) is deposited on silicon surface and then cooled rapidly from room temperature $\left(T_{R} \approx 23{ }^{\circ} \mathrm{C}\right)$ to the desired temperature $T_{s} \approx 3 \pm 1{ }^{\circ} \mathrm{C}$ (the melting temperature of benzene is $5.5^{\circ} \mathrm{C}$ ). The substrate temperature is measured by a $K$-type thermocouple placed in contact with the silicon surface. The chamber is filled with pure $\mathrm{N}_{2}$ gas saturated with water at room temperature. To avoid dust and to saturate the gas, $\mathrm{N}_{2}$ was bubbled in pure water. The gas flow rate was controlled with a flow meter and was kept fixed at $0.2 \mathrm{~L} / \mathrm{min}$. The growth dynamics of the drops was observed with a highresolution charge-coupled device (CCD) camera (COHU, 4910 series, $50 \mathrm{fps}$ ) attached to an optical microscope (Leica, DMRXE, maximum resolution $\approx 2 \mu \mathrm{m}$ ) and recorded on a video recorder. The video images are then analyzed by an image analysis software.

\section{OBSERVATIONS}

When liquid benzene is cooled below its melting point, the frozen surface shows a polygonal type structure with some linear defects along the boundary [see Fig. 1(a), $t$ $=1 \mathrm{~s}$. Similarly to [13], we have also observed during water 
(a)

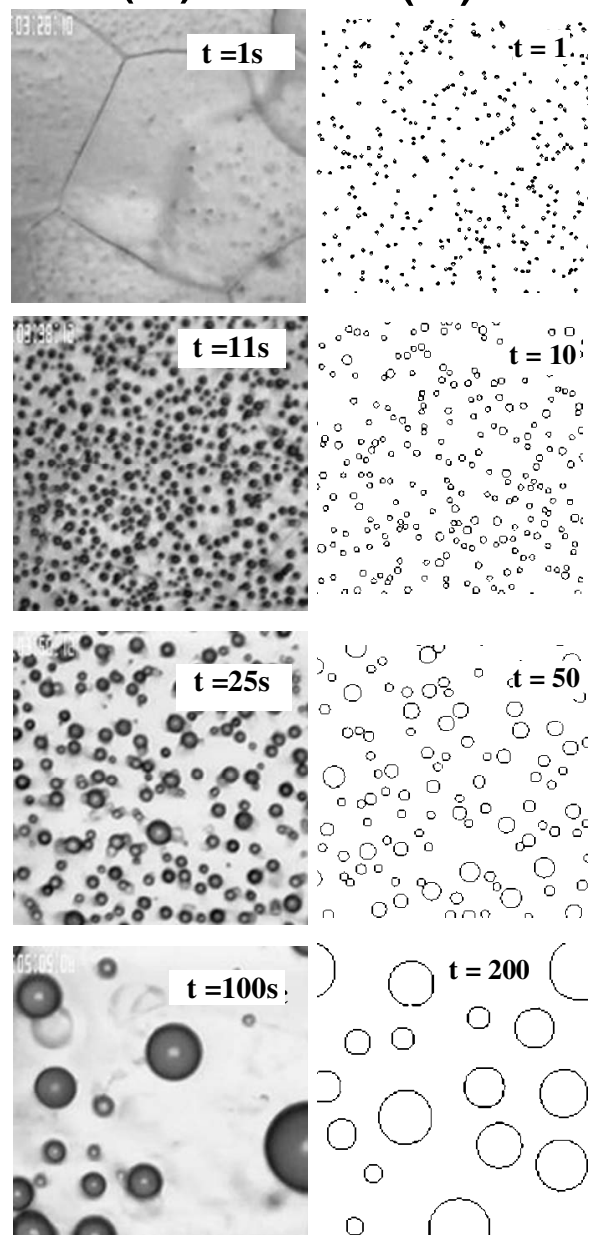

FIG. 1. (a) Evolution of water drop on the surface of benzene (solid) at $3{ }^{\circ} \mathrm{C}$. The images size are $45 \times 45 \mu \mathrm{m}^{2}$. (b) Computer simulation pictures of jumping drops at different time steps. The image size is $450 \times 450$. Initially $10^{5}$ drops of radius $R_{0}=1$ were placed on substrate size of $2500 \times 2500$.

condensation rotational motion of the drop around a point defect that pins the contact line and some times linear motion along the side of polygons. Both these motions were observed during the nucleation of the drops and continue for a few seconds. The most interesting observation is the jumping (hopping) of drops on the substrate. These drop motions continue until the layer of small thickness of solid benzene melt completely.

Figure 2(a) shows a typical picture of instantaneous jumps of many drops in time frame of $20 \mathrm{~ms}$. The earlier positions of the corresponding drops on the substrate are clearly seen. This motion originates in the released of latent heat at the drop perimeter during vapor condensation, which is able to melt the substrate and change the drop-substrate contact angle. As also reported in [13], the drop stay immobile during a time $\tau$ that is proportional to $R$, that is, to the melted substrate mass $\sim 2 \pi R e^{2}$ at drop perimeter. Here $e$ is the thickness of melted substrate around the contact line. The thickness $e$ should be independent of $R$ to fulfill the requirement that $\tau \sim R$. This assumption is indeed supported by the
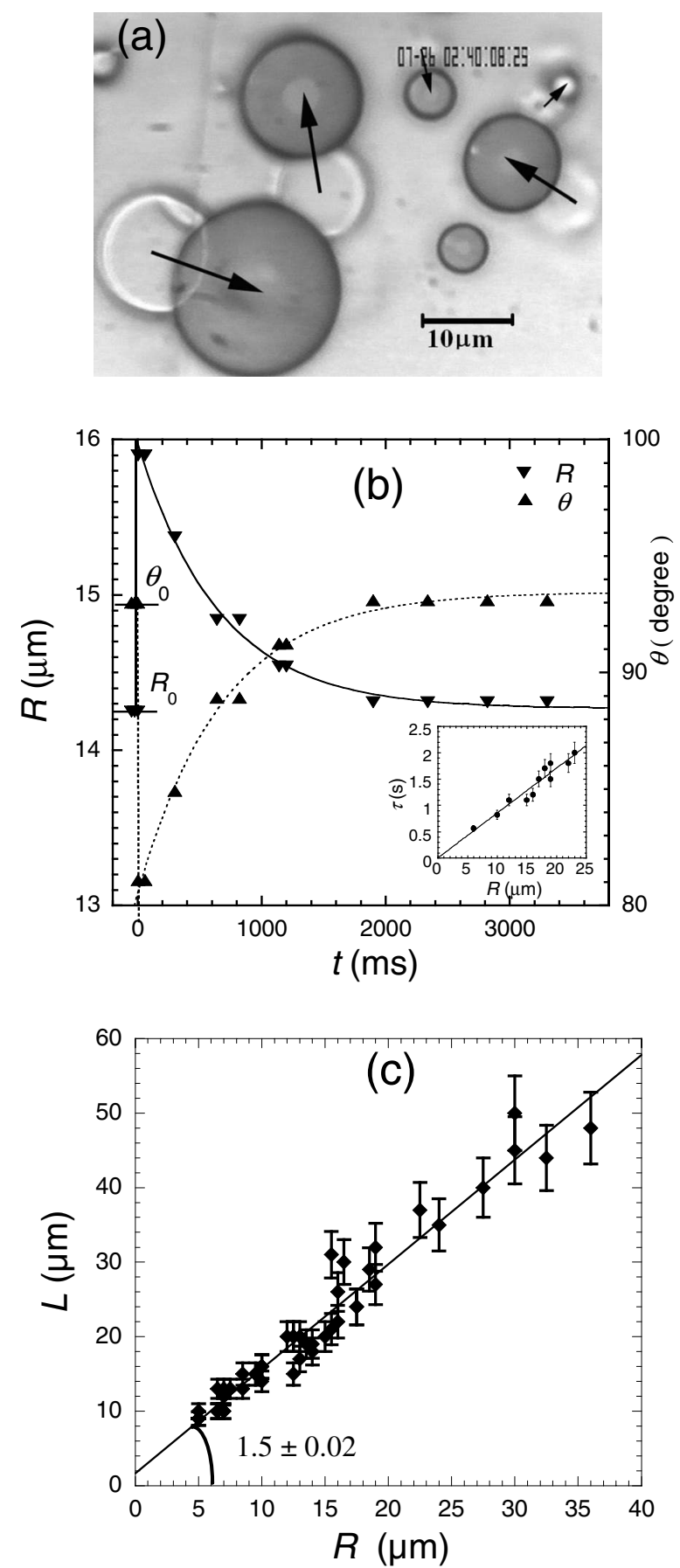

FIG. 2. (a) Microscopic picture of water drops on the surface of solid benzene at $3{ }^{\circ} \mathrm{C}$. The random jumps of drops and their previous positions are clearly seen. (b) Evolution of radius $R$ and contact angle $\theta$ of jumping drop. Inset: time $\tau$ between jumps vs drop radius $R$. The straight line is a fit with inverse slope $(11.7 \pm 0.3) \mu \mathrm{m} \mathrm{s}^{-1}$ (one standard deviation). (c) Drop jump amplitude $(L)$ vs the drop radius $(R)$ in linear scale. The straight line is a fit with slope $1.5 \pm 0.02$ (one standard deviation).

observations. As the latent heat is released symmetrically in the substrate around the drop perimeter, the substrate should melt also symmetrically around the perimeter. Then the layer depth should be equal to the layer thickness. The latter is evidenced by the perimeter print thickness, $e$ [see below and 


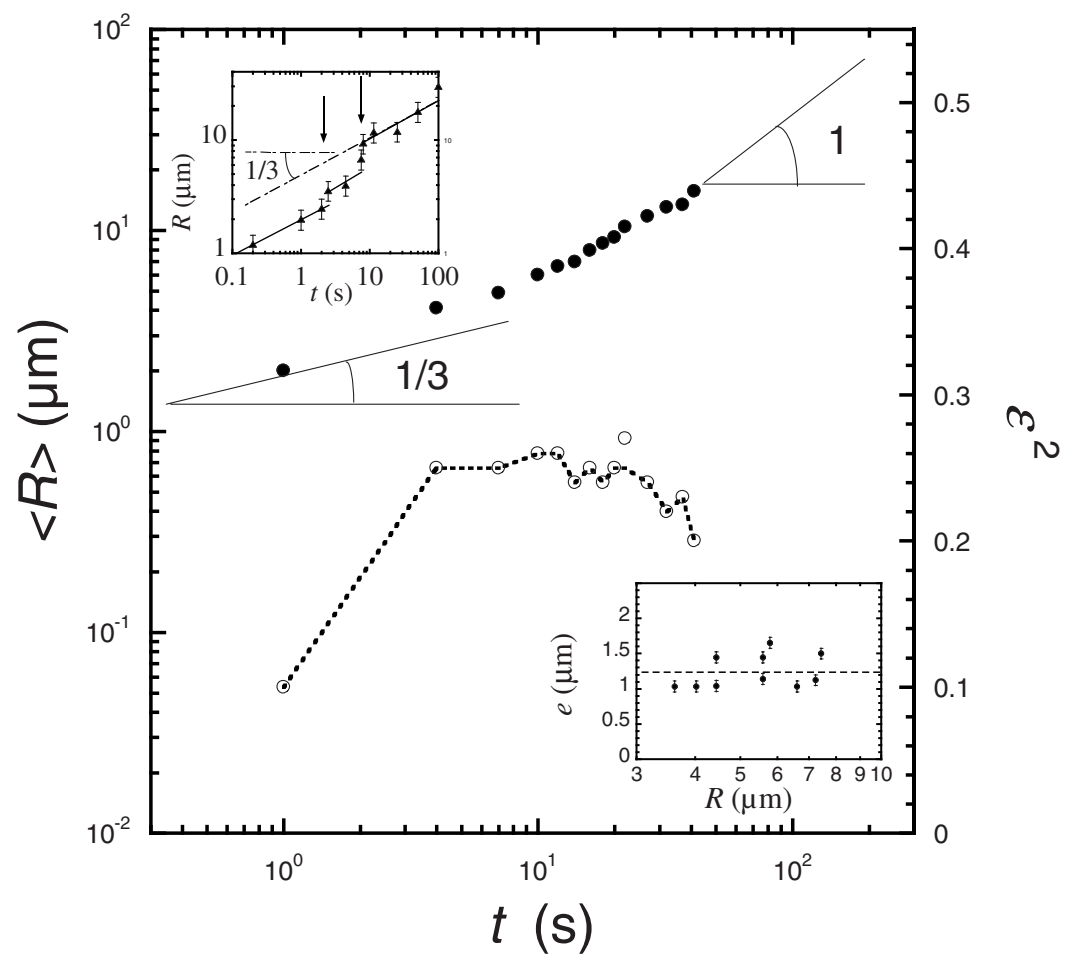

FIG. 3. Evaluation of $\langle R\rangle$ (black circle) and surface coverage $\left(\varepsilon^{2}\right)$ (open circle and interrupted line, a guide for the eyes) for water drops condensed on the surface of solid benzene at $3{ }^{\circ} \mathrm{C}$ (log-log scale). Inset: upper corner is the evolution (log-log scale) of a single drop of radius $(R)$. The arrows indicate drop coalescence. In the lower corner is the print $(e)$ of the melted substrate around the contact line (semilog plot) that is found independent of $R$. The interrupted line is the mean value $(1.24 \pm 0.24) \mu \mathrm{m}$ (error: one standard deviation).

Fig. 2(a)]. In Fig. 3 the plot of $e$ with respect to $R$ shows that $e$ is indeed a constant. The proportionality between $\tau$ and $R$ is shown in the inset of Fig. 2(b): $R / \tau$ $=(11.7 \pm 0.3) \mu \mathrm{m} \mathrm{s}^{-1}$.

Then the drop moves extremely rapidly in a random direction and stops within a distance on order of its radius $R$, leaving apparent the print of its perimeter [Fig. 2(a)], a print that remains visible for $\approx 2 \mathrm{~s}$ and afterward fades gradually. It is also apparent in the video that the print of the drop is smaller than the drop radius just after jump. The drop radius then slowly diminishes to recover its initial value, within a small rise due to condensation. Assuming that the volume $V$ of the drop is constant during the process, which is a good approximation as the condensation rate is low, one can deduce from the evolution of the drop radius the corresponding evolution of the contact angle $\theta$ through,

$$
R^{3}=\frac{3 V}{\pi} \frac{(1+\cos \theta) \sin \theta}{(1-\cos \theta)(2+\cos \theta)} .
$$

The corresponding evolutions are shown in Fig. 2(b), for a drop of initial radius $R=14.25 \mu \mathrm{m}$ and $\theta=94.7^{\circ}$. This value is obtained from the Young's equation by using the surface tension $(\gamma)$ of liquid benzene against water $(36.9 \mathrm{mN} / \mathrm{m}$ at $\left.6{ }^{\circ} \mathrm{C}[19]\right)$, benzene at $3{ }^{\circ} \mathrm{C}(30.32 \mathrm{mN} / \mathrm{m}$ [19]) and water at $3{ }^{\circ} \mathrm{C}(74.9 \mathrm{mN} / \mathrm{m})$. The contact angle drops suddenly by $\approx 10^{\circ}$ when the substrate melts. It results a change in the capillary force balance that thrusts the drop in a random direction. The drop stops when the benzene liquid layer beneath freezes and then its shape slowly relaxes to equilibrium. This mechanism was suggested by Katz [20]. The process is limited by the contact line motion at small capillary number, similar to the one encountered after two drop coalescence [21-23]. In Fig. 2(b), the drop radius relaxes expo- nentially with a relaxation time $(700 \pm 60) \mathrm{ms}$ (one standard deviation). This time has to be compared with the capillary time $R(\eta / \gamma)$ ( $\eta$ is the dynamic viscosity, $\gamma$ is the drop liquidvapor surface tension). One finds a ratio on order of $10^{7}$, which, as outlined in [21-23], means that the triple line dissipation indeed controls the relaxation. This relaxation corresponds to very small Reynolds numbers, typically $\approx 10^{-4}$ for drops of $10 \mu \mathrm{m}$ radius with water kinematic viscosity $\approx 10^{-6} \mathrm{~m}^{2} \mathrm{~s}^{-1}$.

The amplitude of the jump is $1.5 R[$ Fig. 2(c)] and the time between two successive jumps is proportional to $R$ as $\tau$ $=R / \alpha$ [Fig. 2(b), inset], with $\alpha=(11.7 \pm 0.3) \mu \mathrm{m} \mathrm{s}^{-1}$ (one standard deviation), a value similar to what was found on cyclohexane $\alpha=(12.5 \pm 0.3) \mu \mathrm{m} \mathrm{s}^{-1}$ [13] (one standard deviation). We have also attempted to look into the details of the jumps with a faster camera (1000 fps) but failed to see the details of the jump. The upper limit of initial acceleration, $a$, can be estimated as follows. For a capillary force $2 \pi R \gamma$, the acceleration $a=2 \pi R \gamma /\left(2 / 3 \pi R^{3} \rho\right)=3 \gamma / \rho R^{2}$, where $\rho$ is the drop volumic mass with water, $\gamma$ $\approx 73$ dyne $/ \mathrm{cm}, \quad \rho \approx 1 \mathrm{~g} \mathrm{~cm}^{-3}$, the acceleration $a \approx 2.2$ $\times 10^{8} \mathrm{~cm} \mathrm{~s}^{-2}$ and the typical time $t_{0}$ for a jump over $R$ $=10 \mu \mathrm{m}$ is then on order of $2 \mu \mathrm{s}$. This time is indeed much smaller than that of the fast camera limit. The Reynolds number for the jumping drop can be estimated as $\mathrm{Re}$ $=a t_{0} R / v \approx 58$, with $\nu=0.85 \times 10^{-6} \mathrm{~m}^{2} \mathrm{~s}^{-1}$ the liquid benzene kinematic viscosity. This large value means that the flow below the drop might be nonlaminar. However, a detailed description of the drop motion is out of the scope of the present study.

\section{DROP PATTERN GROWTH}

The growth dynamics is very fast, due to the many coalescence events resulting from the jumps, and is completed 
in a couple of minutes. Figure 3 shows a typical evolution. The data are averaged over 800-900 condensed drops at the early stage, while at the late stages the data are averaged over less drops (30-40 drops) since the coalescence reduces the number of drops. When the surface coverage, $\varepsilon^{2}$, is low (typically $<0.15)$, coalescences are inexistent and the average drop radius $\langle R\rangle \sim t^{\beta}$, with $\beta=1 / 3$, as expected for a single drop growth in steady state of condensation where the drop volume increases as time. As the surface coverage increases, to a maximum value of 0.2 (value noticeably much smaller than the value $\sim 0.56$ classically observed on planar surfaces without jumping) [15,24], coalescence becomes more efficient and the usual rescaled growth law $\langle R\rangle \sim t^{1}$ is ultimately recovered. Between these two limiting behaviors an apparent growth law with mean (crossover) exponent $\approx 0.5$ is observed. Also in Fig. 3 (inset) is shown a single drop growth. The evolution between coalescence is in $t^{1 / 3}$, as expected.

\section{COMPUTER SIMULATION}

The growth process was started by randomly placing $10^{5}$ drops of initial radius $R_{0}=1$ unit, on the substrate of size $L$ $\times L(=2500 \times 2500$ units $)$ without overlapping. At each time step, the radius of each drop was increased according to the law $R \sim t^{1 / 3}$ that corresponds to single drop growth The new radius of each drop after every time step was calculated as $R_{n+1}=R_{n}\left(t_{n+1} / t_{n}\right)^{1 / 3}$, where $t_{n+1}$ and $t_{n}$ are the times at $(n$ $+1)$ th and $n$th steps, respectively. The jumps of the drops are incorporated in the simulation as follows. To each drop added on the substrate a counter with value zero is assigned and the time between two successive jumps $\tau=R(t) / 11.7$ is calculated for each drop. In order to fit the experimental situation, the above values were varied randomly within $20 \%$. Then at each time for every drop the counter is increased by one time unit and $\tau$ is calculated. When the counter of any drop exceeds or becomes equal to its $\tau$ value the drop is given a displacement of amplitude $1.5 R$ with random direction and again its counter is set to zero. As above for $\tau$ the displacement is varied randomly within $20 \%$ around its mean value. In case of coalescence (touch or overlap) of two or more $(p)$ drops, the counter of resultant drop is set to zero and its radius $R=\left(R_{1}^{3}+R_{2}^{3}+\ldots R_{p}^{3}\right)^{1 / 3}$ and $\tau$ is calculated. This new drop is placed at the center of mass of the coalescing drops and again checked for further overlap, and the process is repeated until there is no overlap. Periodic boundary conditions are also incorporated. In Fig. 1(b) is shown the drop pattern evolution. A small section of the total substrate is shown in the figure. It can be noticed that experimental and simulation pictures are qualitatively in agreement.

In Fig. 4 are reported the evolution of the pattern average radius and the surface coverage. The classical case where the drops do not move is reported for sake of comparison. All results were averaged over 20 runs. One notes that the numerical simulation data are in agreement with the experimental results as described above.

It is observed that for both jumping and no jumping drops, the very first data points concerning $\langle R\rangle$ and $\varepsilon^{2}$ are the
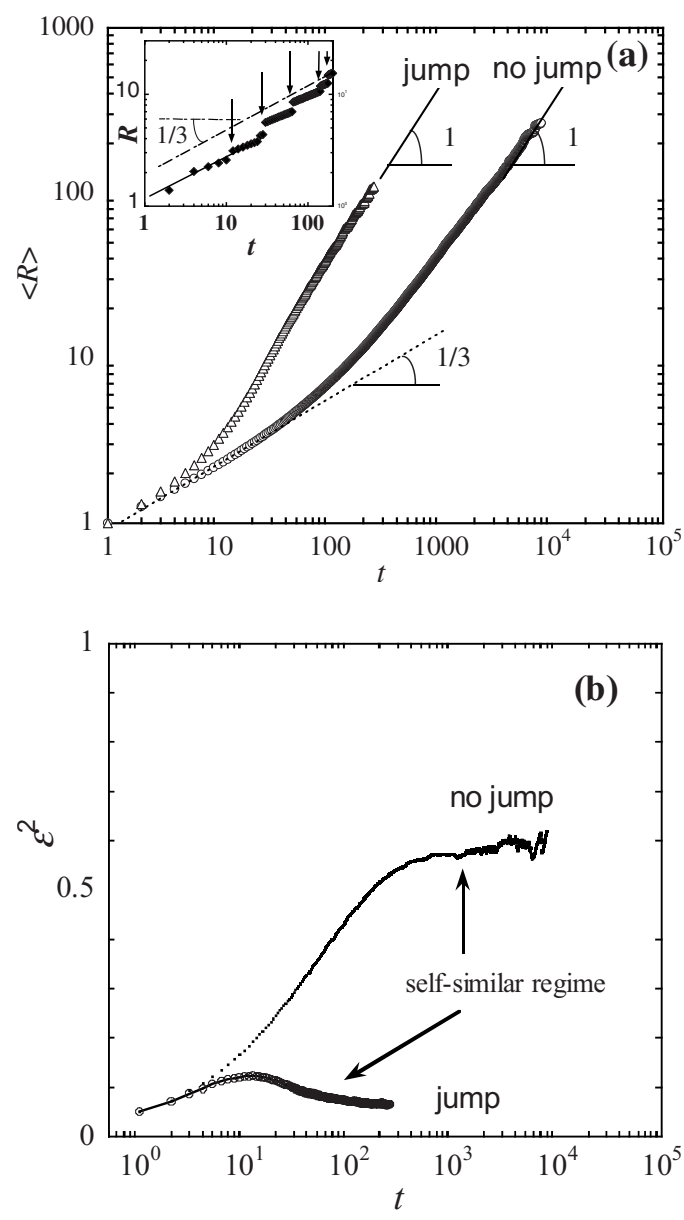

FIG. 4. (a) Log-log plot of $\langle R\rangle$ vs time $t$ for both jump and no-jump case showing the initial stages where coalescence is negligible ( $1 / 3$ exponent, low surface coverage) and final stages dominated by coalescence (exponent unity, high surface coverage). Inset: evolution (log-log scale) of a single drop of radius $(R)$. The arrows indicate drop coalescence. (b) Evolution of surface coverage, $\varepsilon^{2}$, for jump and no-jump case.

same at early time. They corresponds to small surface coverage and negligible coalescence events and give a power law growth with exponent $\beta=1 / 3$. For both jumping and no jumping drops, the limiting behavior at large surface coverage and high number of coalescence events correspond to the same growth law $\langle R\rangle \sim t^{1}$, with however a much smaller limiting surface coverage $(\sim 0.07)$ for jumping drops than for no jumping drops $(\sim 0.6)$. This is due to the fact that the range of drop interaction is markedly larger for the jumping drops. The interaction distance, $\xi$, for collision is on order $\langle R\rangle$ for no-jump, and on order $\langle R\rangle+L=2.5\langle R\rangle$ for jumping drops (Fig. 5). Then the ratio of surface coverage jump/no jump should be in the ratio $(1 / 2.5)^{2}=0.16$, which in effect approximately corresponds to what is observed (no jump: 0.6; jump: $\sim 0.1)$.

In the crossover regime, the surface coverage for jumping drops becomes smaller than for jumping drops, reaches a maximum $(\sim 0.12)$ and then diminishes to saturate at the above limiting value $\sim 0.07$. The value of the surface coverage is a balance between a condensation-induced increase and a coalescence-induced decrease. In the crossover regime, 


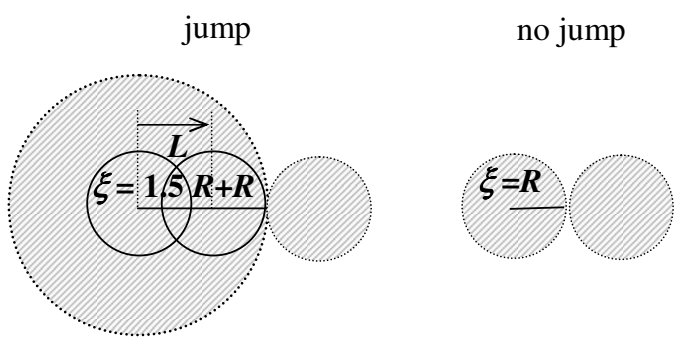

FIG. 5. Schematics showing the difference between jumping and no jumping drop collision with other drop(s) within the interaction distance $\xi$

there is a mix up between the tendency to very low surface coverage due to enhanced jump-induced coalescence events that will dominate at late times and the tendency to a larger surface coverage due to the growth of the drop radius. The different dynamics of both evolution leads to the observed maximum.

\section{CONCLUSION}

This experimental and numerical study of the growth dynamics of water drops condensing on a surface tension active substrate (solid benzene near its melting point) then appears to be not markedly different from that on a simple planar substrate. The interaction distance is simply rescaled by the jump distance. The acceleration of growth due to enhanced coalescence occurs, however, for surface coverage much less than a simple substrate. One also notices that such jumping condensing drops are an extraordinary model of microsteam engines, working however in an isothermal cycle instead of the classical adiabatic cycle (which cannot be used at such micrometer scale where heat diffuses too rapidly).

\section{ACKNOWLEDGMENTS}

R.D.N. acknowledges for the support of a Marie Curie Action within the 7th European Community Framework Program. M.D.K acknowledges the support from The Academy of Finland through its Centre of Excellence (COMP) and TransPoly Consortium grants and P.B.S acknowledges BCUD, Pune University for support. We thank S. Berkowicz for a critical reading.
[1] L. R. Glicksman and A. W. Hunt, Int. J. Heat Mass Transfer 15, 2251 (1972).

[2] R. D. Narhe, M. D. Khandkar, K. P. Adhi, A. V. Limaye, S. R. Sainkar, and S. B. Ogale, Phys. Rev. Lett. 86, 1570 (2001).

[3] D. Beysens, C. M. Knobler, and H. Schaffar, Phys. Rev. B 41, 9814 (1990).

[4] R. S. Singh, C. A. Grimes, and E. C. Dickey, Mater. Res. Innovations 5, 178 (2002).

[5] M. Haupt, S. Miller, R. Sauer, and K. Thonke, J. Appl. Phys. 96, 3065 (2004).

[6] A. Boker, Y. Lin, K. Chiapperini, R. Horowitz, M. Thompson, V. Carreon, T. Xu, C. Abetz, H. Skaff, A. D. Dinsmore, T. Emrick, and T. Russell, Nature Mater. 3, 302 (2004).

[7] D. Beysens, M. Muselli, V. Nikolayev, R. Narhe, and I. Milimouk, Atmos. Res. 73, 1 (2005).

[8] L. Rayleigh, Nature (London) 86, 416 (1911); J. Aitken, ibid. 86, 516 (1911); T. J. Baker, Philos. Mag. 44, 752 (1922).

[9] A. Steyer, P. Guenoun, D. Beysens, and C. M. Knobler, Phys. Rev. A 44, 8271 (1991).

[10] A. V. Limaye, R. D. Narhe, A. M. Dhote, and S. B. Ogale, Phys. Rev. Lett. 76, 3762 (1996).

[11] P. Meakin, Rep. Prog. Phys. 55, 157 (1992).

[12] S. Ulrich, S. Stoll, and E. Pefferkorn, Langmuir 20, 1763 (2004).

[13] A. Steyer, P. Guenoun, and D. Beysens, Phys. Rev. Lett. 68,
64 (1992)

[14] V. A. Kuz, Phys. Rev. E 49, 1246 (1994).

[15] J. L. Viovy, D. Beysens, and C. M. Knobler, Phys. Rev. A 37, 4965 (1988).

[16] B. J. Briscoe and K. P. Galvin, J. Phys. D: Appl. Phys. 23, 422 (1990).

[17] T. M. Rogers, K. R. Elder, and R. C. Desai, Phys. Rev. A 38, 5303 (1988).

[18] D. Fritter, C. M. Knobler, D. Roux, and D. Beysens, J. Stat. Phys. 52, 1447 (1988).

[19] Handbook of Surface and Colloid Chemistry, 3rd ed., edited by K. S. Birdi (CRC Press, Boca Raton, FL, 2008) The value of surface tension $(\gamma)$ of benzene at $3{ }^{\circ} \mathrm{C}$ is obtained from a linear fit in the temperature range -13 to $227^{\circ} \mathrm{C}$ of the data are taken from http://en.wikipedia.org/wiki/Surface_tension.

[20] J. I. Katz, Phys. Rev. E 49, 914 (1994).

[21] C. Andrieu, D. A. Beysens, V. S. Nikolayev, and Y. Pomeau, J. Fluid Mech. 453, 427 (2002).

[22] D. A. Beysens and R. D. Narhe, J. Phys. Chem. B 110, 22133 (2006).

[23] R. Narhe, D. Beysens, and V. S. Nikolayev, Langmuir 20, 1213 (2004).

[24] D. Beysens and C. M. Knobler, Phys. Rev. Lett. 57, 1433 (1986). 